\title{
A Characterization of Strongly Locally Incentive Compatible Planning Procedures with Public Goods
}

\author{
JEAN-JACQUES LAFFONT \\ Université des Sciences Sociales de Toulouse \\ and \\ ERIC MASKIN \\ Massachusetts Institute of Technology
}

\begin{abstract}
This paper provides a systematic study of planning procedures with public goods in which local truthful revelation of preferences is a dominant strategy. These procedures are said to be strongly locally individually incentive compatible (SLIIC). We first characterize the (time invariant) continuously differentiable planning procedures that are SLIIC. Then, we study properties such as balancedness, cheatproofness with respect to coalitions, neutrality, individual rationality and we point out the connection with the MDP procedures.
\end{abstract}

\section{INTRODUCTION}

Planning with public goods was studied by Drèze and de la Vallée Poussin (1971) and Malinvaud (1972) in a class of dynamic procedures called the MDP processes. In these procedures, each consumer reports his marginal rates of substitution between public goods and a private good at each instant. The planning bureau uses this information to alter the allocation of public goods and to make transfers of private good. Over time, the plan converges to a Pareto optimum. Moreover, along the way, the utility of each consumer continually increases; i.e. the procedure is individually rational. Champsaur (1976) showed that the class of MDP procedures is "neutral" or "unbiased"; that is, that any individually rational Pareto optimum is the limit point of a member of this class (see Champsaur, Drèze and Henry (1977) for a comprehensive study of stability and of existence of solutions in such procedures).

One important question about MDP processes concerns the incentive for truthful revelation of marginal rates of substitution. Drèze and de la Vallée Poussin (1971) showed that truthful revelation is a local ${ }^{1}$ maximin strategy and consequently also globally maximin. They also observed that at the stopping point of a process, revelation of true marginal rates of substitution forms a Nash equilibrium. Malinvaud (1971) suggested that MDP procedures would converge even if agents "lied" along the way. Indeed, Roberts (1979) proved that if, at each instant, consumers report their Nash equilibrium strategies of the local revelation game (by the local revelation game, we mean that consumers report so as to maximize the instantaneous increase of utility; Nash equilibrium is unique, but the equilibrium strategies are untruthful except at the stopping point ${ }^{2}$ ), 
the procedure still converges to a Pareto optimum, although at a slower speed than under truthful revelation.

Modelling consumers' behaviour by Nash equilibrium implicitly entails one of two alternative assumptions. Either one assumes that consumers know each other's preferences and so can directly calculate the Nash equilibrium, or one supposes that equilibrium is reached through an iterative adjustment procedure.

One way of avoiding both assumptions is to devise a procedure ensuring that at each instant truthful revelation is a dominant strategy (i.e. is optimal regardless of what other consumers do) for the consumer.

Green and Laffont (1979) devised planning procedures with this incentive property but these were neither individually rational nor balanced. ${ }^{3}$ Using the differential approach of Laffont and Maskin (1980), Fugigaki and Sato (1980) exhibited a class of generalized MDP procedures which are balanced and for which truthful behavior is a locally dominant strategy. One member of this class, moreover, is individually rational; consequently this individually rational procedure converges to a Pareto optimum (see Section 4 below).

In this paper we provide a systematic study of planning procedures with public goods in which local truthful revelation of preferences is a dominant strategy. These procedures are said to be strongly locally individually incentive compatible (SLIIC).

In Section 2 we set up a model with one private and one public good ${ }^{4}$ and define our terms. In Section 3 we completely characterize the continuously differentiable planning procedures that are SLIIC. We give necessary and sufficient conditions for the transfers to be balanced at each instant. Finally, we show that there exist no twice continuously differentiable planning procedure which is proof from manipulation by coalitions. Section 3 characterizes the class of SLIIC, balanced, individually rational procedures that converge to Pareto optima for the case of two consumers. This class is very large, and in fact is neutral. Yet it contains only one member of the MDP family.

When the number of agents is greater than two, no MDP procedure is SLIIC. However, as with two consumers, the class of SLIIC procedures that are Pareto optimal, balanced, and individually rational is large. In Section 4 we characterize this class (assuming differentiability) for an arbitrary number of consumers.

\section{THE MODEL}

We consider economies with $N$ consumers and two commodities: one private good available from initial endowments and one pure public good which can be produced from the private good.

Each consumer $i$ is characterized by a smooth strictly concave utility function $U^{i}\left(x^{i}, y\right)$, defined on the non-negative orthant of $\boldsymbol{R}^{2}$, where $x^{i}$ denotes the consumption of private good and $y$ denotes the level of public good, and by a positive endowment $w^{i}$ of private good.

Moreover

$$
\begin{gathered}
U_{x}^{i} \equiv \frac{\partial U^{i}}{\partial x^{i}}\left(x^{i}, y\right)>0 \quad \text { for any } x^{i}>0, y>0 \\
U_{x}^{i}(0, y)=\infty \quad \text { for any } y \geqq 0 \\
U_{y}^{i} \equiv \frac{\partial U^{i}}{\partial y}\left(x^{i}, y\right) \geqq 0 \quad \text { for } x^{i} \geqq 0, y \geqq 0 \\
\Pi^{i}\left(x^{i}, y\right) \equiv \frac{\partial U^{i}}{\partial y}\left(x^{i}, y\right) / \frac{\partial U^{i}}{\partial x^{i}}\left(x^{i}, y\right) \\
\Pi^{i}(0, y)=0 \quad \text { for any } y \geqq 0 .
\end{gathered}
$$


The production possibilities are described by a smooth cost function $G(y)$ such that

$$
\begin{gathered}
\frac{d G}{d y}(y) \equiv \gamma(y)>0 \\
\frac{d^{2} G}{d y^{2}}(y)>0 \text { for any } y \geqq 0 .
\end{gathered}
$$

we assume that for all $\left\{x^{i}\right\}$ such that $\sum x^{i}=\sum w^{i}$

$$
\sum_{i=1}^{N} \Pi^{i}\left(x^{i}, 0\right)-\gamma(0)>0,
$$

so that positive levels of the public good are always socially desirable.

Definition 1. A planning procedure is an $N$-tuple of strategy spaces $S^{1} \times \cdots \times S^{N}$ and functions $Y(\cdot), X^{1}(\cdot), \ldots, X^{N}(\cdot)$ from $S^{1} \times \cdots \times S^{N}$ to $R^{N+1}$ such that at each instant $t$

$$
\begin{gathered}
\frac{d y}{d t}=\dot{y}=Y\left(s^{1}(t), \ldots, s^{N}(t)\right) \\
\frac{d x^{i}}{d t}=\dot{x}^{i}=X^{i}\left(s^{1}(t), \ldots, s^{N}(t)\right) \quad i=1, \ldots, N
\end{gathered}
$$

where

$$
s(t) \equiv\left(s^{1}(t), \ldots, s^{N}(t)\right) \in S^{1} \times \cdots \times S^{N}
$$

is an $\mathrm{N}$-tuple of announced strategies at instant $t$.

(1) specifies the rate of change of the quantity of public good; (2) describes the rates of change of the private good allocations. The procedure starts at time 0 , when $y(0)=0$ and $x^{i}(0)=w^{i}, i=1, \ldots, N$.

As well as considering it as a dynamic system, we can view a planning procedure as a static game in which each agent announces a global strategy $\left(s^{i}(\cdot)\right)$, and where the outcome is a stationary point of the dynamic system (1) and (2). However we know from the incentives literature (Hurwicz (1972) and others) that there exist no procedure for which consumers have dominant strategies whose equilibrium outcomes are always Pareto optimal. To obtain positive results we must therefore weaken the incentives requirement if Pareto optimality is to be maintained. We shall do this by assuming that consumers are myopic; i.e. we suppose that they maximize their instantaneous pay off-the rate of change of their utility level, $d U^{i} / d t$.

Given this type of behaviour, we study the class of planning procedures for which, at each instant, the optimal strategy of each consumer is a dominant strategy.

Observe that

$$
\frac{d U^{i}}{d t}\left(x^{i}(t), y(t)\right)=\frac{\partial U^{i}}{\partial x^{i}}\left(x^{i}(t), y(t)\right)\left[\Pi^{i}\left(x^{i}(t), y(t)\right) Y(s(t))+X^{i}(s(t))\right] .
$$

Thus, under the myopia assumption, each consumer maximizes

$$
\Pi^{i}\left(x^{i}(t), y(t)\right) Y(s(t))+X^{i}(s(t)) .
$$

At each instant, a consumer acts as if he had a linear utility function defined by $\Pi^{l}$.

From Green and Laffont ((1979) theorems 4-7) we know that, in searching for a procedure with instantaneous dominant strategies there is no loss of generality in assuming that a consumer's strategy space coincides with the space of marginal rates of substitution. 
In this case, a strategy (for consumer $i$ ) is just the announcement of a marginal rate of substitution, $\psi^{i}(t)$. Equivalently, consumer $i$ can announce a net marginal rate of substitution, $\theta^{i}(t)$, defined as follows: Let $\lambda^{i} \geqq 0, i=1, \ldots, N, \sum_{i=1}^{N} \lambda^{i}=1$, be ex-ante defined shares in the marginal cost of the public good.

Then

$$
\theta^{i}(t) \equiv \theta^{i}\left(x^{i}(t), y(t)\right) \equiv \psi^{i}\left(x^{i}(t), y(t)\right)-\lambda^{i} \gamma(y(t))
$$

is the announced net marginal rate of substitution $\left(\theta^{i}(t) \in R\right)$.

Let

$$
\hat{\theta}^{i}(t) \equiv \Pi^{i}\left(x^{i}(t), y(t)\right)-\lambda^{i} \gamma(y(t))
$$

be the true (net) marginal rate of substitution.

$$
\begin{aligned}
\frac{d U^{i}}{d t}\left(x^{i}(t), y(t)\right) & =\frac{\partial U^{i}}{\partial x^{i}}\left(x^{i}(t), y(t)\right)\left[\Pi^{i}(t) \dot{y}(t)+\dot{x}^{i}(t)\right] \\
& =\frac{\partial U^{i}}{\partial x^{i}}\left(x^{i}(t), y(t)\right)\left[\hat{\theta}^{i}(t) \dot{y}(t)+\dot{x}^{i}(t)+\lambda^{i} \gamma(t) \dot{y}(t)\right] \\
& \equiv \frac{\partial U^{i}}{\partial x^{i}}\left(x^{i}(t), y(t)\right)\left[\hat{\theta}^{i}(t) \dot{y}(t)+\dot{\tau}^{i}(t)\right]
\end{aligned}
$$

with

$$
\dot{\tau}^{i}(t)=\dot{x}^{i}(t)+\lambda^{i} \gamma(t) \dot{y}(t)
$$

and $\tau^{i}(0)=0$.

The $\dot{\tau}^{i}$ are transfers of private good beyond those needed to finance the public good. They play a major role in inducing the right incentives. For convenience, we shall henceforth consider $U^{i}(\cdot), \Pi^{i}(\cdot), \theta^{i}(\cdot)$ as functions of $\tau^{i}$ and $y$ instead of $x^{i}$ and $y$.

We can summarize the above discussion by the following definitions:

Definition 2. A local direct revelation planning procedure is a planning procedure for which the strategy of each agent at each instant is an announced (net) marginal rate of substitution $\theta^{i}(t)$, i.e. $S^{i}=\mathbb{R}$, and

$$
\begin{aligned}
\dot{y} & =Y\left(\theta^{1}(t), \ldots, \theta^{N}(t)\right)^{5} \\
\dot{x}^{i} & =X^{i}\left(\theta^{1}(t), \ldots, \theta^{N}(t)\right) \quad i=1, \ldots, N
\end{aligned}
$$

or

$$
\dot{\tau}^{i}=T^{i}\left(\theta^{1}(t), \ldots, \theta^{N}(t)\right)
$$

Announcing his true net marginal rate of substitution $\hat{\theta}^{i}$ is a dominant strategy for consumer $i$ at a given instant if

$$
\hat{\theta}^{i} Y\left(\hat{\theta}^{i}, \theta^{-i}\right)+T\left(\hat{\theta}^{i}, \theta^{-i}\right) \geqq \hat{\theta}^{i} Y\left(s^{i}, \theta^{-i}\right)+T^{i}\left(s^{i}, \theta^{-i}\right),
$$

for all $s^{i}$ and $\theta^{-i 6}$.

Definition 3. A strongly locally individually incentive compatible (SLIIC) planning procedure is a local direct revelation planning procedure for which revelation of the true (net) marginal rate of substitution is a dominant strategy at each instant for every consumer.

Definition 4. A strongly locally coalitionally incentive compatible (SLCIC) planning procedure is a SLIIC planning procedure such that revelation of the true (net) 
marginal rates of substitution is a dominant strategy at each instant for every coalition of consumers.

Before studying SLIIC planning procedures, we require a few more definitions.

Definition 5. A planning procedure is $n$-times continuously differentiable or $C^{n}$ iff the functions $Y(\cdot), T^{1}(\cdot), \ldots, T^{N}(\cdot)$ are $C^{n}$.

Definition 6. A planning procedure is balanced iff:

$$
\sum_{i=1}^{N} \dot{\tau}^{i}(t)=0 \quad \forall t \geqq 0 .
$$

Note that if a procedure is balanced, then $\sum_{i=1}^{N} \tau^{i}=0$ at each instant.

An allocation $\left(y, \tau^{1}, \ldots, \tau^{N}\right)$ is Pareto optimal iff

$$
\begin{gathered}
\sum_{i=1}^{N} \hat{\theta}^{i}\left(\tau^{i}, y\right)=0 \\
\sum_{i=1}^{N} \tau^{i}=0 .
\end{gathered}
$$

(4) is the Lindahl-Samuelson condition, and (5) is the feasibility constraint for the private good. The necessity and sufficiency of (4) and (5) for Pareto optimality follows from the convexity of preferences and technology, non-satiation in the private good, and the boundary conditions $\Pi^{i}(0, y)=0$ and $\sum_{i=1}^{N} \Pi^{i}\left(w^{i}, 0\right)-\gamma(0)>0$.

Definition 7. A balanced planning procedure is Pareto optimal iff its stationary points are Pareto optimal allocations; i.e.

$$
\left.\begin{array}{rl}
Y\left(\theta^{1}, \ldots, \theta^{N}\right) & =0 \\
T^{i}\left(\theta^{1}, \ldots, \theta^{N}\right) & =0 \quad i=1, \ldots, N
\end{array}\right\} \Leftrightarrow \quad \sum_{i=1}^{N} \theta^{i}=0 \quad \sum_{i=1}^{N} \tau^{i}=0
$$

Definition 8. A planning procedure is convergent iff the solutions of the dynamic system (3) converge to its stationary points.

Definition 9. A planning procedure is dynamically efficient iff it is balanced, Pareto optimal and convergent.

A useful property for proving the dynamic efficiency of a procedure is individual rationality. An allocation is individually rational if no consumer considers it worse than his initial endowment.

Definition 10. A planning procedure is individually rational iff:

$$
\forall i=1, \ldots, N \quad \frac{d U^{i}}{d t}\left(\tau^{i}(t), y(t)\right) \geqq 0 \quad \forall t \geqq 0 .
$$

Definition 11. A class of planning procedures is neutral or unbiased iff each individually rational Pareto optimal allocation is the limit point of a member of the class.

\section{CHARACTERIZATION OF SLIIC PLANNING PROCEDURES}

Our first step will be to characterize planning procedures that are SLIIC, without worrying about efficiency, balance, or individual rationality. 
Theorem 1. A $C^{1}$-planning procedure is SLIIC iff:

(i) $Y(\theta)$ is weakly increasing in each component

(ii) $T^{i}(\theta)=-\int_{0}^{\theta^{i}} s\left(\partial / \partial s^{i}\right) Y\left(s, \theta^{-i}\right) d s+h^{i}\left(\theta^{-i}\right)$ where $h^{i}(\cdot)$ is an arbitrary function of $\theta^{-i}, i=1, \ldots, N$.

Proof. At each instant the maximand of consumer $i$ is

$$
\hat{\theta}^{i} Y\left(\theta^{i}, \theta^{-i}\right)+T^{i}\left(\theta^{i}, \theta^{-i}\right) .
$$

A necessary condition for $\theta^{i}=\hat{\theta}^{i}$ to be a dominant strategy is that $\hat{\theta}^{i}$ be a solution of the first order condition of the consumer's maximization problem.

$$
\hat{\theta}^{i} \frac{\partial Y}{\partial \theta^{i}}\left(\hat{\theta}^{i}, \theta^{-i}\right)+\frac{\partial T^{i}}{\partial \theta^{i}}\left(\hat{\theta}^{i}, \theta^{-i}\right)=0 \text { for any } \theta^{-i} \in R^{N-1} .
$$

Since we seek procedures for which the truth is always a dominant strategy, (6) must hold for any $\hat{\theta}^{i} \in R$. Consequently we have an identity:

$$
\theta^{i} \frac{\partial Y}{\partial \theta^{i}}\left(\theta^{i}, \theta^{-i}\right)+\frac{\partial T^{i}}{\partial \theta^{i}}\left(\theta^{i}, \theta^{-i}\right) \equiv 0 \quad \text { for any }\left(\theta^{i}, \theta^{-i}\right) \in R^{N} .
$$

Integrating (7), we derive

$$
T^{i}(\theta)=-\int_{0}^{\theta^{i}} s \frac{\partial Y}{\partial s^{i}}\left(s, \theta^{-i}\right) d s+h^{i}\left(\theta^{-i}\right)
$$

To see that $Y(\cdot)$ must be weakly increasing let $\theta^{i}, \tilde{\theta}^{i}$ be any two values of consumer $i$ 's marginal rate of substitution. For $\theta^{i}$ to be a dominant strategy when it is the truth, we must have in particular

$$
\theta^{i} Y\left(\theta^{i}, \theta^{-i}\right)+T^{i}\left(\theta^{i}, \theta^{-i}\right) \geqq \theta^{i} Y\left(\tilde{\theta}^{i}, \theta^{-i}\right)+T^{i}\left(\tilde{\theta}^{i}, \theta^{-i}\right) .
$$

Similary when $\tilde{\theta}^{i}$ is the truth

$$
\tilde{\theta}^{i} Y\left(\tilde{\theta}^{i}, \theta^{-i}\right)+T^{i}\left(\tilde{\theta}^{i}, \theta^{-i}\right) \geqq \tilde{\theta}^{i} Y\left(\theta^{i}, \theta^{-i}\right)+T^{i}\left(\theta^{i}, \theta^{-i}\right) .
$$

Adding (9) and (10) yields:

$$
\left(\theta^{i}-\tilde{\theta}^{i}\right)\left[Y\left(\theta^{i}, \theta^{-i}\right)-Y\left(\tilde{\theta}^{i}, \theta^{-i}\right)\right] \geqq 0 .
$$

Hence $Y$ is weakly increasing.

Observe that this last argument does not require differentiability. When differentiability holds, this condition can be derived from the local second order condition of the above maximization problem. Indeed the second derivative is

$$
\hat{\theta}^{i} \frac{\partial^{2} Y}{\left(\partial \theta^{i}\right)^{2}}\left(\theta^{i}, \theta^{-i}\right)+\frac{\partial^{2} T^{i}}{\left(\partial \theta^{i}\right)^{2}}\left(\theta^{i}, \theta^{-i}\right)
$$

Using (7) and evaluating (11) at $\theta^{i}=\hat{\theta}^{i}$ we have:

$$
-\frac{\partial Y}{\partial \theta^{i}}\left(\hat{\theta}^{i}, \theta^{-i}\right)
$$

The second-order condition becomes:

$$
-\frac{\partial Y}{\partial \theta^{i}}\left(\hat{\theta}^{i}, \theta^{-i}\right) \leqq 0 \text { or } \frac{\partial Y}{\partial \theta^{i}}\left(\hat{\theta}^{i}, \theta^{-i}\right) \geqq 0 \text { for any }\left(\hat{\theta}^{i}, \theta^{-i}\right) \in R^{N} .
$$

It remains to check that these necessary conditions are indeed sufficient; i.e. that $\theta^{i}=\hat{\theta}^{i}$ is a global maximum of consumer $i$ 's maximization problem. We must verify that 


$$
\hat{\theta}^{i} Y\left(\hat{\theta}^{i}, \theta^{-i}\right)+T^{i}\left(\hat{\theta}^{i}, \theta^{-i}\right) \geqq \hat{\theta}^{i} Y\left(\theta^{i}, \theta^{-i}\right)+T^{i}\left(\theta^{i}, \theta^{-i}\right) \text { for any }\left(\theta^{i}, \theta^{-i}\right) \in R^{N} .
$$

Using (8) and integrating by parts, we can rewrite (12) as

$$
\left(\hat{\theta}^{i}-\theta^{i}\right) Y\left(\theta^{i}, \theta^{-i}\right) \leqq \int_{\theta^{i}}^{\hat{\theta}^{i}} Y\left(s^{i}, \theta^{-i}\right) d s^{i},
$$

which holds from the weak monotonicity of $Y\left(\theta^{i}, \theta^{-i}\right)$. \|

Given this complete characterization of SLIIC planning procedures, we can now impose additional desiderata.

Theorem 2. Suppose that $Y(\theta)$ is weakly increasing and $C^{N}$. There exists a balanced SLIIC $C^{N}$-planning procedure with public good allocation rule $Y(\theta)$ iff:

$$
\frac{\partial^{N}}{\partial \theta^{1} \cdots \partial \theta^{N}} Y(\theta) \equiv 0 .
$$

Proof. If transfers are balanced,

$$
\sum_{i=1}^{N} T^{i}(\theta) \equiv 0 .
$$

From (8), (13) is equivalent to

$$
\sum_{i=1}^{N}-\int_{0}^{\theta^{i}} s^{i} \frac{\partial Y}{\partial s^{i}}\left(s^{i}, \theta^{-i}\right) d s^{i}+h^{i}\left(\theta^{-i}\right) \equiv 0 .
$$

Differentiating with respect to $\theta^{1}, \ldots, \theta^{N},(14)$ yields:

$$
\left(\sum_{i=1}^{N} \theta^{i}\right) \frac{\partial^{N}}{\partial \theta^{1} \cdots \partial \theta^{N}} Y(\theta) \equiv 0
$$

which implies:

$$
\frac{\partial^{N}}{\partial \theta^{1} \cdots \partial \theta^{N}} Y(\theta) \equiv 0 \quad \text { since } Y(\cdot) \text { is } C^{N} .
$$

Conversely, if (16) holds, we can reintegrate it to construct $h^{i}(\cdot)$ functions such that transfers are balanced. \|

Theorem 3. A balanced SLIIC planning procedure is Pareto optimal iff:

$$
Y(\theta)=0 \quad \Leftrightarrow \quad \sum_{i=1}^{N} \theta^{i}=0 .
$$

Proof. Obvious.

Theorem 4. There exists no $C^{2}$-SLCIC Pareto optimal planning procedure.

Proof. Consider a coalition of two agents, say agents 1 and 2 . They choose $\left(\theta^{1}, \theta^{2}\right)$ to maximize the following objective function ${ }^{7}$

$$
\left(\hat{\theta}^{1}+\hat{\theta}^{2}\right) Y(\theta)-\int_{0}^{\theta^{1}} s^{1} \frac{\partial Y}{\partial s^{1}}\left(s^{1}, \theta^{-1}\right) d s^{1}-\int_{0}^{\theta^{2}} s^{2} \frac{\partial Y}{\partial s^{2}}\left(s^{2}, \theta^{-2}\right) d s^{2}+h^{1}\left(\theta^{-1}\right)+h^{2}\left(\theta^{-2}\right) .
$$


Hence, the first order conditions are:

$$
\begin{aligned}
& \left(\hat{\theta}^{1}+\hat{\theta}^{2}\right) \frac{\partial Y}{\partial \theta^{1}}(\theta)-\theta^{1} \frac{\partial Y}{\partial \theta^{1}}(\theta)-\int_{0}^{\theta^{2}} s^{2} \frac{\partial^{2} Y}{\partial s^{2} \partial \theta^{1}}\left(s^{2}, \theta^{-2}\right) d s^{2}+\frac{\partial h^{2}}{\partial \theta^{1}}\left(\theta^{-2}\right)=0 \\
& \left(\hat{\theta}^{1}+\hat{\theta}^{2}\right) \frac{\partial Y}{\partial \theta^{2}}(\theta)-\theta^{2} \frac{\partial Y}{\partial \theta^{2}}(\theta)-\int_{0}^{\theta^{1}} s^{1} \frac{\partial^{2} Y}{\partial s^{1} \partial \theta^{2}}\left(s^{1}, \theta^{-1}\right) d s^{1}+\frac{\partial h^{1}}{\partial \theta^{2}}\left(\theta^{-1}\right)=0 .
\end{aligned}
$$

For $\left(\hat{\theta}^{1}, \hat{\theta}^{2}\right)$ to be a joint dominant strategy for the coalition, (17) and (18) must hold as identities when $\theta^{1}=\hat{\theta}^{1}, \theta^{2}=\hat{\theta}^{2}$ from the same argument as used in Theorem 1.

In particular (17) yields:

$$
\left(\theta^{1}+\theta^{2}\right) \frac{\partial Y}{\partial \theta^{1}}(\theta)-\theta^{1} \frac{\partial Y}{\partial \theta^{1}}(\theta)-\int_{0}^{\theta^{2}} s^{2} \frac{\partial^{2} Y}{\partial s^{2} \partial \theta^{1}}\left(s^{2}, \theta^{-2}\right) d s^{2}+\frac{\partial h^{2}}{\partial \theta^{1}}\left(\theta^{-2}\right) \equiv 0 .
$$

Differentiating (19) with respect to $\theta^{2}$ gives:

$$
\left(\theta^{1}+\theta^{2}\right) \frac{\partial^{2} Y}{\partial \theta^{1} \partial \theta^{2}}+\frac{\partial Y}{\partial \theta^{1}}-\theta^{1} \frac{\partial^{2} Y}{\partial \theta^{1} \partial \theta^{2}}-\theta^{2} \frac{\partial^{2} Y}{\partial \theta^{2} \partial \theta^{1}} \equiv 0
$$

or

$$
\frac{\partial Y}{\partial \theta^{1}} \equiv 0
$$

Similarly (18) yields $\partial Y / \partial \theta^{2} \equiv 0$.

Considering all coalitions of two agents successively, we conclude that $Y(\theta)$ must be a constant $K$. But then $Y(\theta)$ clearly does not satisfy the condition of Theorem 3 . Therefore the procedure is not Pareto optimal.

We next seek to construct dynamically efficient SLIIC planning procedures. This objective is easily reached if one can design Pareto optimal, balanced procedures that are individually rational. We show below that this is possible.

\section{PARETO OPTIMALITY, INDIVIDUAL RATIONALITY, AND NEUTRALITY WHEN $\mathrm{N}=2$}

Because the arguments are especially simple, we begin with the case $N=2$. In Section 5 we extend to arbitrary $N$.

A complete characterization of autonomous ${ }^{9}$ SLIIC, Pareto optimal, balanced, individually rational $C^{2}$-planning procedures is provided in the following theorem.

Theorem 5. A two-person $C^{2}$ planning procedure is SLIIC, Pareto optimal, balanced and individually rational iff

(i) $Y(\theta)=\Delta\left(\theta^{1}\right)-\Delta\left(-\theta^{2}\right), \Delta$ strictly increasing and continuously differentiable

(ii) $T^{1}(\theta)=-\int_{0}^{\theta^{1}} s^{1}\left(d / d s^{1}\right) \Delta\left(s^{1}\right) d s^{1}+\int_{0}^{\theta^{2}} s^{2}(d / d s)^{2} \Delta\left(-s^{2}\right) d s^{2}$.

$$
T^{2}(\theta)=-T^{1}(\theta) \text {. }
$$

Proof. From Theorem 2, balance requires:

$$
Y(\theta) \equiv Y^{1}\left(\theta^{1}\right)+Y^{2}\left(\theta^{2}\right) .
$$

Incentive compatibility requires:

$$
\frac{d Y^{1}}{d \theta^{1}}\left(\theta^{1}\right) \geqq 0 \quad \frac{d Y^{2}}{d \theta^{2}}\left(\theta^{2}\right) \geqq 0
$$


From Theorem 3, Pareto optimality requires:

$$
Y^{1}\left(\theta^{1}\right) \equiv-Y^{2}\left(-\theta^{1}\right) .
$$

Let denote $\Delta(\cdot) \equiv Y^{1}(\cdot)$. Then, $Y(\theta)=\Delta\left(\theta^{1}\right)-\Delta\left(-\theta^{2}\right)$.

To avoid the existence of stationary points different from the Pareto optima, $\Delta(\cdot)$ must be strictly increasing.

From Theorem 1,

$$
\begin{aligned}
& T^{1}(\theta)=-\int_{0}^{\theta^{1}} s^{1} \frac{d \Delta}{d s^{1}}\left(s^{1}\right) d s^{1}+h^{1}\left(\theta^{2}\right) \\
& T^{2}(\theta)=-\int_{0}^{\theta^{2}} s^{2} \frac{d \Delta}{d s^{2}}\left(-s^{2}\right) d s^{2}+h^{2}\left(\theta^{1}\right) .
\end{aligned}
$$

To ensure balance, we must have

$$
\begin{aligned}
& T^{1}(\theta)=-\int_{0}^{\theta^{1}} s^{1} \frac{d \Delta}{d s}\left(s^{1}\right) d s^{1}+\int_{0}^{\theta^{2}} s^{2} \frac{d \Delta}{d s^{2}}\left(-s^{2}\right) d s^{2}+K \\
& T^{2}(\theta)=-\int_{0}^{\theta^{2}} s^{2} \frac{d \Delta}{d s^{2}}\left(-s^{2}\right) d s^{2}+\int_{0}^{\theta^{1}} s^{1} \frac{d \Delta}{d s^{1}}\left(s^{1}\right) d s^{1}-K,
\end{aligned}
$$

for some constant $K$.

It remains to show that with $Y$ and $T^{i}$ so defined the procedures are individually rational if and only if $K=0$.

$$
\frac{d U^{i}}{d t}=\frac{\partial U^{i}}{\partial x^{i}}\left(x^{i}(t), y(t)\right)\left[\theta^{i} Y(\theta)+T^{i}(\theta)\right]
$$

with $\partial U^{i} / \partial x^{i}>0$ and, after integration by parts,

$$
\begin{aligned}
\theta^{1} Y(\theta)+T^{1}(\theta) & =\theta^{1}\left[\Delta\left(\theta^{1}\right)-\Delta\left(-\theta^{2}\right)\right]-\int_{0}^{\theta^{1}} s^{1} \frac{\mathrm{d} \Delta}{d s^{1}}\left(s^{1}\right) d s^{1}+\int_{0}^{\theta^{2}} s^{2} \frac{d \Delta}{d s^{2}}\left(-s^{2}\right) d s^{2}+K \\
& =\int_{-\theta^{2}}^{\theta^{1}} \Delta(s) d s-\left(\theta^{1}+\theta^{2}\right) \Delta\left(-\theta^{2}\right)+K \geqq 0
\end{aligned}
$$

where, from the monotonicity of $\Delta(\cdot)$, the last expression is non-negative for all $\left(\theta^{1}, \theta^{2}\right)$ if and only if $K \geqq 0$.

Similarly,

$$
\theta^{2} Y(\theta)+T^{2}(\theta)=\left(\theta^{1}+\theta^{2}\right) \Delta\left(\theta^{1}\right)-\int_{-\theta^{2}}^{\theta^{1}} \Delta(s) d s-K,
$$

which is non-negative for all $\left(\theta^{1}, \theta^{2}\right)$ if and only if $K \leqq 0$. $\|$

Thanks to individual rationality, the dynamic efficiency of the SLIIC procedures defined in Theorem 5 is easily proved. Our assumptions ensure that the feasible set is bounded. Taking $U^{1}(\cdot)+U^{2}(\cdot)$ as a Lyapunov function, the procedures are quasi-stable in the Uzawa sense; that is, any limit point of the trajectory is a stationary point. Stability then follows since, from the strict convexity of preferences and technology, stationary points are Pareto optimal and there is a unique Pareto optimum that Pareto dominates all points on the trajectory. ${ }^{8}$ 
Remark 1. If $\Delta(s) \equiv s, Y(\theta)=\theta^{1}+\theta^{2}$,

$$
\begin{aligned}
& T^{1}(\theta)=-\frac{\left(\theta^{1}\right)^{2}}{2}+\frac{\left(\theta^{2}\right)^{2}}{2}=-\theta^{1} \dot{y}+\frac{1}{2} \dot{y}^{2} \\
& T^{2}(\theta)=-\frac{\left(\theta^{2}\right)^{2}}{2}+\frac{\left(\theta^{1}\right)^{2}}{2}=-\theta^{2} \dot{y}+\frac{1}{2} \dot{y}^{2} .
\end{aligned}
$$

this is the MDP procedure with equal surplus sharing.

By varying $\Delta(\cdot)$, a whole family of SLIIC dynamically efficient planning procedures can be constructed.

For example take $\Delta(s)=s^{3}$; then $Y(\theta)=\left(\theta^{1}\right)^{3}+\left(\theta^{2}\right)^{3}$

$$
\begin{aligned}
& T^{1}(\theta)=-\frac{3}{4}\left(\theta^{1}\right)^{4}+\frac{3}{4}\left(\theta^{2}\right)^{4} \\
& T^{2}(\theta)=-\frac{3}{4}\left(\theta^{2}\right)^{4}+\frac{3}{4}\left(\theta^{1}\right)^{4} .
\end{aligned}
$$

Remark 2. Since incentive compatibility is a "closed" property (that is, inequality (9) is weak), even a non-differentiable point-wise limit of a differentiable sequence $\left\{\Delta^{n}\right\}$ definies a SLIIC procedure. To ensure that only Pareto optima are stationary points of the limit procedure, the limit function must be strictly increasing.

As Champsaur and Rochet (1981) showed, it is a simple matter to see that the class $C$ of two-person, individually rational, dynamically efficient SLIIC planning procedures is neutral. Consider the portion of the Pareto frontier that Pareto dominates the initial endowment. There are two extreme points on this curve, namely the ones where either consumer 1 (point $\alpha$ ) or consumer 2 (point $\beta$ ) is no better off than with his initial endowment. To demonstrate neutrality, one need only show that members of $C$ come arbitrarily close to attaining point $\alpha$. By symmetry, the same is true of point $\beta$. Then, by continuity the entire portion of the Pareto frontier is attainable. Champsaur and Rochet (1981) pointed out that if in Theorem 5 we take $\Delta(s)=e^{a s}$, then as a tends to infinity, the corresponding stationary point converges to $\alpha$.

\section{SLIIC PLANNING PROCEDURES WHEN N $>2$}

In this section we generalize the characterization theorems of the previous section to arbitrary $N$. We begin (Theorem 6) by stating a characterization result that does not impose individual rationality. We then do the same (Theorem 7) in the case where balance is not imposed. Finally in Theorem 8 we prove the counterpart of Theorem 5 for $N>2$.

Theorem 6. A $C^{N}$-planning procedure is SLIIC, Pareto optimal, balanced iff:

(a) $Y(\theta)$ is weakly increasing

(b) $\left(\partial^{N} Y / \partial \theta^{1} \cdots \partial \theta^{N}\right)(\theta) \equiv 0$

(c) $Y(\theta)=0 \Leftrightarrow \sum_{i=1}^{N} \theta^{i}=0$

(d) $T^{i}(\theta)=-\int_{0}^{\theta^{1}} s \partial Y / \partial s^{i}\left(s, \theta^{-i}\right) d s+h^{i}\left(\theta^{-i}\right) \quad i=1, \ldots, N$.

where the functions $h^{i}(\cdot)$ are chosen so that $\sum_{i=1}^{N} T^{i}(\theta)=0$ (such a choice is possible from Theorem 2).

Proof. Obvious from previous theorems.

Remark 3. For $N \geqq 3$, the MDP procedures are not SLIIC. For an MDP procedure, $Y(\theta)=\sum_{i=1}^{N} \theta^{i}$. In this case incentive compatible transfers must be of the form $T^{i}(\theta)=$ 
$-\left(\theta^{i}\right)^{2} / 2+h^{i}\left(\theta^{-i}\right)$. The transfers of the MDP procedure are, for $i=1, \ldots, N$,

$$
\begin{aligned}
& -\theta^{i}\left(\sum_{j=1}^{N} \theta^{j}\right)+\delta^{i}\left(\sum_{j=1}^{N} \theta^{j}\right)^{2}, \\
= & -\left(1-\delta^{i}\right)\left(\theta^{i}\right)^{2}+\delta^{i}\left(\sum_{j \neq i} \theta^{j}\right)^{2}+\left(2 \delta^{i}-1\right) \theta^{i} \sum_{j \neq i} \theta^{j}
\end{aligned}
$$

where $\sum \delta^{i}=1$ and, for all $i, \delta^{i}>0$.

Thus the required separability of $T^{i}$ obtains only when $2 \delta^{i}-1=0$. This is possible for all consumers if and only if $N=2$. follows:

We can characterize Pareto optimal, individually rational SLIIC procedures as

Theorem 7. A $C^{N}$-planning procedure is SLIIC, Pareto optimal, and individually rational if and only if

(a) $Y(\theta)$ is weakly increasing

(b) $Y(\theta)=0 \Leftrightarrow \sum_{i=1}^{N} \theta^{i}=0$

(c) $T^{i}(\theta)=-\theta^{i} Y(\theta)+\int_{-\left(\sum_{j \neq i} \theta^{i}\right)}^{\theta^{i}} Y\left(s, \theta^{-j}\right) d s$.

Proof. From Theorem 6 we know that

$$
\begin{aligned}
T^{i}(\theta) & =-\int_{0}^{\theta^{i}} s \frac{\partial Y}{\partial s^{i}}\left(s, \theta^{-i}\right) d s+h^{i}\left(\theta^{-i}\right) \\
& =-\theta^{i} Y(\theta)+\int_{0}^{\theta^{i}} Y\left(s, \theta^{-i}\right) d s+h^{i}\left(\theta^{-i}\right) .
\end{aligned}
$$

But

$$
\int_{0}^{\theta^{i}} Y\left(s, \theta^{-i}\right) d s+h^{i}\left(\theta^{-i}\right)=\int_{-\sum_{j \neq i} \theta^{j}}^{\theta^{i}} Y\left(s, \theta^{-i}\right) d s+\tilde{h}^{i}\left(\theta^{-i}\right) .
$$

Since $Y(\cdot)$ is weakly increasing and $\theta^{i} \geqq \sum \theta^{j}$ (from the boundary condition $\sum \Pi^{i}\left(x^{i}, 0\right)-\gamma(0)$, we know that $\sum \theta^{i}>0$ when the procedure starts. Since the process stops as soon as $\sum \theta^{i}=0$, we conclude that $\sum \theta^{i} \geqq 0$ always),

$$
\int_{-\sum_{j \neq i} \theta^{j}}^{\theta^{i}} Y\left(s, \theta^{-i}\right) d s \geqq 0 .
$$

For any $\theta^{-i}, Y\left(\theta^{i}, \theta^{-i}\right)$ and this last integral are zero for $\theta^{i}=-\sum_{j \neq 1} \theta^{j}$ so that individual rationality implies $\tilde{h}^{i}\left(\theta^{-i}\right) \geqq 0$. However when $\theta^{i}=\sum_{i \neq i} \theta^{j}, Y(\theta)=0$, and so the sum of the transfers equals $\sum \tilde{h}^{i}\left(\theta^{-i}\right)$. Since $\theta^{i}=\sum_{j \neq i} \theta^{j}$ corresponds to a Pareto optimum, the sum of the transfers must vanish. Therefore $\tilde{h}^{i}\left(\theta^{-i}\right) \equiv 0, i=1, \ldots, N$. $\|$

Remark 4. Note that in the proof of Theorem 7 we establish the individual rationality of the procedures only for $\theta$ such that $\sum \theta^{i} \geqq 0$, in particular on equilibrium paths starting from $y=0$. This is in contrast with Theorem 5, whose procedures are individually rational everywhere.

If, in addition to the requirements of Theorem 7 , we require that the planning procedure be balanced, then, according to the Theorem we can conclude that

$$
J(\theta) \underset{\text { Def. }}{=}-Y(\theta) \sum_{i=1}^{N} \theta^{i}+\sum_{i=1}^{N} \int_{-\sum_{j \neq i} \theta^{j}}^{\theta^{i}} Y\left(s, \theta^{-i}\right) d s \equiv 0 .
$$

Although (20) and (a)-(c) of theorem 7 are together necessary and sufficient for the procedure to be SLIIC, Pareto optimal, individually rational, and balanced, (20) is not 
an especially transparent condition. To put it in somewhat more useful form, let us suppose that $Y(\theta)$ is an analytic function. Then (20) holds if and only if all partial derivatives of $J(\theta)$ vanish for $\theta \in \theta^{*}=\left\{\tilde{\theta} \mid \sum \tilde{\theta}^{i}=0\right\}$.

Now

$$
\begin{aligned}
\frac{\partial J}{\partial \theta^{1}}(\theta)= & -Y(\theta)-\frac{\partial Y}{\partial \theta^{1}}(\theta) \sum_{i=1}^{N} \theta^{i}+Y(\theta)+\sum_{i \neq 1} Y\left(-\sum_{j \neq i} \theta^{i}, \theta^{-i}\right) \\
& +\sum_{i \neq 1} \int_{-\sum_{j \neq i} \theta^{\theta^{i}}}^{\theta^{i}} \frac{\partial Y}{\partial \theta^{1}}\left(s, \theta^{-i}\right) d s \\
= & 0 \quad \text { if } \theta \in \theta^{*} \text { and (b) of Theorem } 7 \text { holds }
\end{aligned}
$$

and

$$
\begin{aligned}
\frac{\partial^{2} J}{\partial\left(\theta^{1}\right)^{2}}(\theta)= & -\frac{\partial Y}{\partial \theta^{1}}(\theta)-\frac{\partial^{2} Y}{\partial\left(\theta^{1}\right)^{2}}(\theta) \sum \theta^{i}+\sum_{i \neq 1} \frac{\partial Y}{\partial \theta^{1}}\left(-\sum_{j \neq 1} \theta^{j}, \theta^{-i}\right) \\
& +\sum_{i \neq 1} \int_{-\sum_{j \neq i} \theta^{i}}^{\theta^{i}} \frac{\partial^{2} Y}{\partial\left(\theta^{1}\right)^{2}}\left(s, \theta^{-i}\right) d s .
\end{aligned}
$$

therefore, if $N>2,(21)$ implies that, since $\left(\partial^{2} J / \partial\left(\theta^{1}\right)^{2}\right)(\theta)$ vanishes for $\theta \in \theta^{*}$,

$$
\frac{\partial Y}{\partial \theta^{1}}(\theta)=0 \quad \text { for } \theta \in \theta^{*}
$$

Continuing iteratively, we find that, for given $k$, if for all $t<k,\left(\partial^{t} Y / \partial\left(\theta^{1}\right)^{t}\right)(\theta)=0$ for $\theta \in \theta^{*}$, then

$$
\begin{aligned}
\frac{\partial^{k+1} J}{\partial\left(\theta^{1}\right)^{k+1}}(\theta)= & -\sum_{i=1}^{N} \theta^{i} \frac{\partial^{k+1} Y}{\partial\left(\theta^{1}\right)^{k+1}}(\theta)-K \frac{\partial^{k} Y}{\partial\left(\theta^{1}\right)^{k}}(\theta)+\sum_{i \neq 1} \frac{\partial^{k} Y}{\partial\left(\theta^{1}\right)^{k}}\left(-\sum_{j \neq i} \theta^{j}, \theta^{-i}\right) \\
& +\sum_{i \neq 1} \int_{-\sum_{j \neq i} \theta^{\theta^{i}}}^{\theta^{i}} \frac{\partial^{k+1} Y}{\partial\left(\theta^{r}\right)^{k+1}}\left(s, \theta^{-i}\right) d s .
\end{aligned}
$$

Thus for $k \leqq N-2$ and $\theta \in \theta^{*}$

$$
\frac{\partial^{k} Y}{\partial\left(\theta^{2}\right)^{k}}(\theta)=0 .
$$

Next, observe that for $1<a \leqq N-2$ and $\theta \in \theta^{*}$

$$
\begin{aligned}
\frac{\partial^{a+1} J}{\partial\left(\theta^{1}\right)^{a} \partial \theta^{r}}(\theta)= & -\sum_{i=1}^{N} \theta^{i} \frac{\partial^{a+1} Y}{\partial\left(\theta^{1}\right)^{a} \partial \theta^{r}}(\theta)-\frac{\partial^{a} Y}{\partial\left(\theta^{1}\right)^{a}}(\theta)-(a-1) \frac{\partial^{a} Y}{\partial\left(\theta^{1}\right)^{a-1} \partial \theta^{r}}(\theta) \\
& +\frac{\partial^{a} Y}{\partial\left(\theta^{1}\right)^{a}}(\theta)+\sum_{i \neq 1, r} \frac{\partial^{a} Y}{\partial\left(\theta^{1}\right)^{a}}\left(-\sum_{j \neq i} \theta^{j}, \theta^{-i}\right) \\
& +\sum_{i \neq 1, r} \int_{-\sum_{j \neq \theta^{\prime}} \theta^{\prime}}^{\theta^{i}} \frac{\partial^{a+1} Y}{\partial\left(\theta^{1}\right)^{a} \partial \theta^{r}}\left(s, \theta^{-i}\right) \\
= & -(a-1) \frac{\partial^{a} Y}{\partial\left(\theta^{1}\right)^{a-1} \partial \theta^{r}}(\theta), \text { from (24). }
\end{aligned}
$$

Therefore

$$
\frac{\partial^{a} Y}{\partial\left(\theta^{1}\right)^{a-1} \partial \theta^{r}}(\theta)=U .
$$


Continuing iteratively, suppose that, for any $m<N$ and for all $a_{1}, \ldots, a_{m}$ with $a=$ $\sum_{i=1}^{m} a_{i} \leqq N-2$ and $\sum_{i=2}^{m} a_{i}<k$,

$$
\frac{\partial^{a} Y}{\partial\left(\theta^{1}\right)^{a_{1}} \cdots \partial\left(\theta^{m}\right)^{a_{m}}}(\theta)=0 \quad \text { for } \theta \in \theta^{*} .
$$

Then for $b_{1}, \ldots, b_{m}$ with $b=\sum_{i=1}^{m} b_{i} \leqq N-2$ and $\sum_{i=2}^{m} b_{i}=k$,

$$
\begin{aligned}
\frac{\partial^{b+1} J(\theta)}{\partial\left(\theta^{1}\right)^{b_{1}+1} \partial\left(\theta^{2}\right)^{b_{2}} \cdots \partial\left(\theta^{m}\right)^{b_{m}}}= & -\sum_{i=1}^{N} \theta^{i} \frac{\partial^{b+1} Y(\theta)}{\partial\left(\theta^{1}\right)^{b_{1}+1} \partial\left(\theta^{2}\right)^{b_{2}} \cdots \partial\left(\theta^{m}\right)^{b_{m}}} \\
& -b_{1} \frac{\partial^{b} Y(\theta)}{\partial\left(\theta^{1}\right)^{b_{1}} \cdots \partial\left(\theta^{m}\right)^{b_{m}}} \\
& -\sum_{i=2}^{m}\left(b_{i}-1\right) \frac{\partial^{b} Y(\theta)}{\partial\left(\theta^{1}\right)^{b_{1}+1} \partial\left(\theta^{2}\right)^{b_{2}} \cdots \partial\left(\theta^{i}\right)^{b_{i}-1} \cdots \partial\left(\theta^{m}\right)^{b_{m}}} \\
& +\sum_{r>m} \int_{-\sum_{i \neq *} \theta^{i}}^{\theta^{r}} \frac{\partial^{b+1} Y(\theta)}{\partial\left(\theta^{1}\right)^{b_{1}+1} \partial\left(\theta^{2}\right)^{b_{2}} \cdots \partial\left(\theta^{m}\right)^{b_{m}}} .
\end{aligned}
$$

Therefore

$$
\frac{\partial^{b+1} J(\theta)}{\partial\left(\theta^{1}\right)^{b_{1}+1} \partial\left(\theta^{2}\right)^{b_{2}} \cdots \partial\left(\theta^{m}\right)^{b_{m}}}=-b_{1} \frac{\partial^{b} Y(\theta)}{\partial\left(\theta^{1}\right)^{b_{1}} \cdots \partial\left(\theta^{m}\right)^{b_{m}}} \quad \text { for } \theta \in \theta^{*}
$$

and we obtain

$$
\frac{\partial^{b} Y(\theta)}{\partial\left(\theta^{1}\right)^{b_{1}} \cdots \partial\left(\theta^{m}\right)^{b_{m}}}=0 \text { for } \theta \in \theta^{*} .
$$

Combining (24)-(26) we conclude that (25) holds for all $m$ and $\left\{b_{1} \cdots, b_{m}\right\}$ such that $\sum b_{i} \leqq N-2$.

Condition (26) and its symmetric counterparts are not only necessary for balance but, in conjunction with (a)-(c) of Theorem 7 and (b) of Theorem 6 they are sufficient as well.

Theorem 8. An analytic planning procedure (i.e. one where $Y$ is analytic) is SLIIC, individually rational, Pareto optimal, and balanced if and only if

(a) $Y(\theta)$ is weakly increasing

(b) $Y(\theta)=0$ if and only if $\sum_{i=1}^{N} \theta^{i}=0$

(c) $T^{i}(\theta)=-\theta^{i} Y(\theta)+\int_{-\sum_{j \neq i} \theta^{i}}^{\theta^{i}} Y\left(s, \theta^{-i}\right) d s$

(d) $\left(\partial^{N} Y(\theta) / \partial \theta^{1} \cdots \cdot \partial \theta^{N}\right)(\theta) \equiv 0$.

(e) $\left(\partial^{a} Y(\theta) / \partial\left(\theta^{1}\right)^{a_{1}} \ldots \partial\left(\theta^{N}\right)^{a_{N}}\right)=0$ for all $\left\{a_{1}, \ldots, a_{N}\right\}$ such that $\sum_{i=1}^{N} a_{i}=a \leqq$ $N-2$ and $\theta \in \theta^{*}$.

Proof. We have already demonstrated the necessity of (a)-(e). To see that they are sufficient, consider the case $N=3$; the generalization to $N>3$ is straightforward. From (d), we may write

$$
Y(\theta)=Y^{1}\left(\theta^{2}, \theta^{3}\right)+Y^{2}\left(\theta^{1}, \theta^{3}\right)+Y^{3}\left(\theta^{1}, \theta^{2}\right) .
$$


Therefore

$$
\begin{aligned}
J(\theta)=T^{i}(\theta)= & -\sum_{i=1}^{3} \theta^{i} Y(\theta)+\sum_{i=1}^{3} \int_{-\sum_{j \neq i} \theta^{j}}^{\theta^{i}} Y\left(s, \theta^{-i}\right) d s \\
= & \int_{-\sum_{j \neq 1} \theta^{i}}^{\theta^{1}}\left(Y^{2}\left(s, \theta^{3}\right)+Y^{3}\left(s, \theta^{2}\right)\right) d s+\int_{-\sum_{j \neq 2} \theta^{i}}^{\theta^{2}}\left(Y^{1}\left(s, \theta^{3}\right)+Y^{3}\left(\theta^{1}, s\right)\right) d s \\
& +\int_{-\sum_{j \neq 3} \theta^{i}}^{\theta^{3}}\left(Y^{1}\left(\theta^{2}, s\right)+Y^{2}\left(\theta^{1}, s\right)\right) d s .
\end{aligned}
$$

Observe that for $\theta \in \theta^{*}, J(\theta)=0$. Furthermore,

$$
\begin{aligned}
\frac{\partial J}{\partial \theta_{1}}= & Y^{2}\left(\theta^{1}, \theta^{3}\right)+Y^{3}\left(\theta^{1}, \theta^{2}\right)+Y^{1}\left(-\sum_{j \neq 2} \theta^{j}, \theta^{3}\right)+Y^{3}\left(\theta^{1},-\sum_{j \neq 2} \theta^{j}\right) \\
& +Y^{1}\left(\theta^{2},-\sum_{j \neq 3} \theta^{j}\right)+Y^{2}\left(\theta^{1},-\sum_{j \neq 3} \theta^{j}\right)+\int_{-\sum_{j \neq 2} \theta^{j}} \frac{\partial Y^{3}}{\partial \theta^{1}}\left(\theta^{1}, s\right) d s \\
& +\int_{-\sum_{j \neq 3} \theta^{i}}^{\theta^{3}} \frac{\partial Y^{2}}{\partial \theta^{1}}\left(\theta^{1}, s\right) d s \\
= & \int_{-\sum_{j \neq 2} \theta^{i}}^{\theta^{2}} \frac{\partial Y^{3}}{\partial \theta^{1}}\left(\theta^{1}, s\right) d s+\int_{-\sum_{j \neq 3} \theta^{j}}^{\theta^{3}} \frac{\partial Y^{2}}{\partial \theta^{1}}\left(\theta^{1}, s\right) d s, \text { from (b) } \\
= & \int_{-\sum_{j \neq 2} \theta^{i}}^{\theta^{i}} \frac{\partial Y^{3}}{\partial \theta^{1}}\left(\theta^{1}, s\right) d s+\int_{-\theta^{3}}^{\sum_{j \neq 3} \theta^{i}} \frac{\partial Y^{2}}{\partial \theta^{1}}\left(\theta^{1},-s\right) d s \\
= & \int_{-\sum_{j \neq 2} \theta^{i}}^{\theta^{i}}\left(\frac{\partial Y^{3}}{\partial \theta^{1}}\left(\theta^{1}, s\right)+\frac{\partial Y^{2}}{\partial \theta^{1}}\left(\theta^{1},-s-\theta^{1}\right)\right) d s .
\end{aligned}
$$

But from (e),

$$
\frac{\partial Y^{3}}{\partial \theta^{1}}\left(\theta^{1}, s\right)+\frac{\partial Y^{2}}{\partial \theta^{1}}\left(\theta^{1},-s-\theta^{1}\right)=0 \quad \text { for all } s
$$

Therefore,

$$
\frac{\partial J}{\partial \theta_{1}}(\theta) \equiv 0 .
$$

Similarly

$$
\frac{\partial J}{\partial \theta_{2}}(\theta) \equiv \frac{\partial J}{\partial \theta_{3}}(\theta) \equiv 0
$$

Hence $J(\theta) \equiv 0 . \quad \|$

Let us consider, as one application of Theorem 8 , the class of procedures proposed by Fugigaki and Sato (1981). In this class, the function $Y(\theta)$ takes the form

$$
Y(\theta)=a\left(\sum_{i=1}^{N} \theta^{i}\right)^{b},
$$

where $b$ is a positive integer and

$$
a=c \operatorname{sign}\left(\sum_{i=1}^{N} \theta^{i}\right)^{b-1},
$$

for some positive constant $c$. Clearly all members of the class satisfy conditions (a) and (b) of Theorem 8. Condition (d) is satisfied if and only if $b \leqq N-1$, whereas (a) holds if and only if $b \geqq N-1$. Therefore, as Fugigaki and Sato showed, the only members of 
their class that are SLIIC, Pareto optimal, individually rational and balanced are those where $b=N-1$ (Theorem 8 was established for analytic functions $Y$, but easily extends to functions that are piece-wise analytic).

There are, of course, many procedures, other than these of Fugigaki and Sato, that satisfy the conditions of Theorem 9. For example, in the case $N=3$, set

$$
Y^{1}\left(\theta^{2}, \theta^{3}\right)=\left(\theta^{2}+\theta^{3}\right)^{3} .
$$

For three consumers, condition (e) of Theorem 8 reduces to

$$
\begin{aligned}
& \frac{\partial Y^{1}}{\partial \theta^{2}}\left(\theta^{2}, \theta^{3}\right)+\frac{\partial Y^{3}}{\partial \theta^{2}}\left(\theta^{1}, \theta^{2}\right)=0 \\
& \frac{\partial Y^{1}}{\partial \theta^{3}}\left(\theta^{2}, \theta^{3}\right)+\frac{\partial Y^{2}}{\partial \theta^{1}}\left(\theta^{1}, \theta^{3}\right)=0 \\
& \frac{\partial Y^{2}}{\partial \theta^{1}}\left(\theta^{1}, \theta^{3}\right)+\frac{\partial Y^{3}}{\partial \theta^{1}}\left(\theta^{1}, \theta^{2}\right)=0
\end{aligned}
$$

for $\theta \in \theta^{*}$. From (28) and (29)

$$
\frac{\partial Y^{3}}{\partial \theta^{2}}\left(\theta^{1}, \theta^{2}\right)=-3\left(\theta^{2}+\left(-\theta^{1}-\theta^{2}\right)\right)^{2}=-3\left(\theta^{1}\right)^{2}
$$

Hence

$$
Y^{3}\left(\theta^{1}, \theta^{2}\right)=-3\left(\theta^{1}\right)^{2} \theta^{2}+h\left(\theta^{1}\right) .
$$

From (28) and (30)

$$
\frac{\partial Y^{2}}{\partial \theta^{3}}\left(\theta^{1}, \theta^{3}\right)=-3\left(\theta^{3}+\left(-\theta^{1}-\theta^{3}\right)\right)^{2}=-3\left(\theta^{1}\right)^{2} .
$$

Thus

$$
Y^{2}\left(\theta^{1}, \theta^{2}\right)=-3\left(\theta^{1}\right)^{2} \theta^{3}+K\left(\theta^{1}\right)
$$

From (31)-(33)

$$
-6 \theta^{1}\left(-\theta^{1}-\theta^{2}\right)+K^{\prime}\left(\theta^{1}\right)-6 \theta^{1} \theta^{2}+h^{\prime}\left(\theta^{1}\right)=0 .
$$

Therefore

$$
h^{\prime}\left(\theta^{1}\right)=-K^{\prime}\left(\theta^{1}\right)-6\left(\theta^{1}\right)^{2},
$$

and so

$$
h\left(\theta^{1}\right)=-K\left(\theta^{1}\right)-2\left(\theta^{1}\right)^{3} .
$$

we conclude that

$$
Y(\theta)=\left(\theta^{2}+\theta^{3}\right)^{3}-3\left(\theta^{1}\right)^{2} \theta^{3}-3\left(\theta^{1}\right)^{2} \theta^{2}-2\left(\theta^{1}\right)^{3} .
$$

It can easily be verified that $Y$ defined by (34) vanishes only if $\theta \in \theta^{*} . Y$ is not increasing for all values of $\theta$. However, if we define $Y^{*}$ by (34) where $Y$ is increasing and by some other rule(s) satisfying (a), (b), (d), and (e) where $Y$ is not, the procedure corresponding to $Y^{*}$ will be SLIIC, individually rational, Pareto optimal, and balanced.

This example suggests, and inspection of conditions (d) and (e) of Theorem 8 confirms, that to generate the family of all SLIIC, individually rational, Pareto optimal, and balanced procedures, we can simply vary $Y^{1}\left(\theta^{-1}\right)$ (where $Y(\theta)=\sum Y^{i}\left(\theta^{-i}\right)$ by virtue of $(\mathrm{d})$ ). Once $Y^{1}\left(\theta^{-1}\right)$ is fixed, the other $Y^{i}\left(\theta^{-i}\right)(i \neq 1)$ are determined uniquely by (e). 
Thus the space of procedures is of the same dimension as the space of functions of $Y^{1}\left(\theta^{-1}\right)$. Of course, given $Y^{1}$, we must check that the corresponding $Y$ satisfies (a) and (b).

First version received December 1980; final version accepted July 1982 (Eds.)

\section{NOTES}

1. "Locally maximin" means "maximizes the minimum instantaneous payoff". "Globally maximin" means "maximizes the minimum ultimate payoff". The instantaneous payoff is the gradient of the utility function whereas the ultimate payoff is the utility of the final allocation.

2. Hurwicz (1972) and Roberts (1979) showed that truthful behaviour cannot constitute a global Nash equilibrium. However, Champsaur and Laroque (1980) showed that, if the procedure is truncated at time $T$, then the global Nash equilibrium allocations converge to Lindahl equilibria as $T$ tends to infinity.

3. A planning procedure is individually rational if consumers' utilities are non-decreasing at each instant. The procedure is balanced if the transfers sum to zero at each instant.

4. Generalization to several public goods is straightforward.

5. For simplicity of exposition we do not take into account the constraint that $y$ must remain positive. However, given the boundary condition $\sum \Pi^{i}\left(x^{i}, 0\right)-\gamma(0)>0$, all the procedures in the class we characterize satisfy this constraint automatically.

6. $\theta^{-i}=\left(\theta^{1}, \ldots, \theta^{i-1}, \theta^{i+1}, \ldots, \theta^{N}\right),\left(s^{i}, \theta^{-i}\right)=\left(\theta^{1}, \ldots, \theta^{i-1}, s^{i}, \theta^{i+1}, \ldots, \theta^{N}\right)$.

7. We assume here that agents who form a coalition share their information truthfully. See Laffont and Maskin (1980) for more on coalitional behaviour.

8. See Champsaur, Drèze and Henry (1977) for details.

9. We restrict the analysis to planning procedures defined by time independent functions $Y$ and $T^{\imath}$.

\section{REFERENCES}

CHAMPSAUR, P. (1976), "Neutrality of planning procedures in an economy with public goods", Review of Economic Studies, 43, 293-300.

CHAMPSAUR, P., DREZE, J. and HENRY, C. (1977), "Stability theorems with economic applications", Econometrica, 45, 273-294.

CHAMPSAUR, P. and LAROQUE, G. (1980), "Strategic behavior in decentralized planning procedures" (Document de Travail No. 8, INSEE, Paris).

CHAMPSAUR, P. and ROCHET, J. C. (1981), "On planning procedures which are locally strategy proof" (Document de Travail No. 8109, INSEE, Paris)

DREZE, J. and DE LA VALLEE POUSSIN, D. (1971), "A tâtonnement process for public goods", Review of Economic Studies, 38, 133-150.

FUGIGAKI, Y. and SATO, K. (1981). "Incentives in the generalized MDP procedure for the provision of public goods", Review of Economic Studies, 48, 473-486.

GREEN, J. and LAFFONT, J. J. (1979) Incentives in Public Decision Making, Studies in Public Economics, Vol. 1 (Amsterdam: North Holland).

HURWICZ, L. (1972), "On informationally decentralized systems", in McGuire and Radner (eds.) Decision and Organization: A Volume in Honor of J. Marschak (Amsterdam: North Holland).

LAFFONT, J. J. and MASKIN, E. (1980), "A differential approach to dominant strategy mechanisms", Econometrica, 48, 1507-1520.

MALINVAUD, E. (1971), "A planning approach to the public good problem", Swedish Journal of Economics, 73, 96-112.

MALINVAUD, E. (1972), "Prices for individual consumption, quantity indicators for collective consumption", Review of Economic Studies, 39, 385-405.

ROBERTS, J. (1979), "Incentives in planning procedures for the provision of public goods", Review of Economic Studies, 66, 283-292. 